\title{
Hamstring Foam Roller release and Sole Self Myofascial Release for Improving Hamstring Muscles Flexibility in Participants with Hamstring Shortness
}

\author{
Geun-Woo Kim, PT • Ji-Hyun Lee, PT, $\mathrm{PhD}^{\dagger}$
}

Department of Physical Therapy, Baekseok University

Received: July 27, 2020 / Revised: July 30, 2020 / Accepted: September 3, 2020

(c) 2020 J Korean Soc Phys Med

\section{| Abstract |}

PURPOSE: The current generation has shortened hamstrings due to a sedentary lifestyle, resulting in reduced flexibility of the hamstring and dysfunction. This study was undertaken to compare effects of three different release exercises on hamstring flexibility, in participants with short hamstrings.

METHODS: Totally, 20 subjects having short hamstrings were involved in this study. The inclusion criterion for study participation was active knee extension test (AKET) less than $60^{\circ}$. All participants were subjected to 3 methods: hamstring foam roller release (HFRR), sitting self myofascial release (sitting SMR), and standing self myofascial release (Standing SMR). All participants randomly performed all three methods to avoid bias caused by learning or fatigue. Passive knee extension test (PKET), AKET, finger to floor distance test (FTFT), and pelvic tilting angle test (PTAT) were measured pre- and post-exercises.

RESULTS: The PKET, AKET and FTFT were significantly

Corresponding Author : Ji-Hyun Lee

Jihyun.lee@bu.ac.kr, https://orcid.org/0000-0002-9864-5485

This is an Open Access article distributed under the terms of the Creative Commons Attribution Non-Commercial License (http://creativecommons.org/licenses/by-nc/3.0) which permits unrestricted non-commercial use, distribution, and reproduction in any medium, provided the original work is properly cited. increased after HFRR, sitting SMR, and standing SMR exercise $(\mathrm{p}<.05)$. However, PTAT was not significantly increased after the three exercises ( $p>.05)$. Furthermore, no significant differences were observed between PKET, AKET, FTFT and PTAT subsequent to HFRR, sitting SMR, and standing SMR $(\mathrm{p}>.05)$.

CONCLUSION: Our results indicate that HFRR, sitting SMR and standing SMR were immediately effective in improving hamstring flexibility in participants with short hamstrings.

Key Words: Hamstring muscle, Pelvis, Relaxed, Stretching

\section{Introduction}

The emerging recent years have shown shortened hamstrings in the current population due to the sedentary lifestyle, resulting in reduced flexibility of the hamstring [1]. The hamstring is a muscle that extends the hip joint and flexes the knee joint. Also, it reduces lumbar lordosis with the coupling force of gluteus maximus, rectus abdominis, and external oblique, which are involved in posterior tilting of the pelvis [2].

To increase flexibility of hamstrings, previous researches have applied various methods, including static stretching using loads [3] and hamstring foam roller release (HFRR) 
[4]. The most commonly used method of improving hamstring flexibility is static stretching. However, it has been reported that static stretching as a part of warm-up before exercise reduces the power and exercise performance [7]. Hence, foam roller massage is applied to increase the range of motion (ROM). According to a previous study in healthy adults, HFRR resulted in significant increase in the range of knee joint motion, as compared to before intervention [4].

The fascia is a connective tissue that surrounds all nerves, blood vessels, and muscle fibers in the body. The bones, muscles, and organs are inter-connected to form a large network throughout the body [5]. When considering the fascia connections of the body, it has been suggested that tensions occurring in particular areas may be detrimental by effectively reducing the overall flexibility (The theory of anatomy trains) [6]. One study, conducted over 8 weeks, investigated the effect of the method applied to stretch the fascia line, for improving Taekwondo side kick performance [7]. They reported that the method of stretching the fascia line was effective in improving the angular speed of the side kick motion of Taekwondo [7]. Another study applied the theory of anatomy trains; the participant was asked to flex the hip and knee in a supine position, allowing the soles to touch the floor, thereby allowing the examiner to relax the fascia by sole stretching. The tension of the erector spine muscles was found to be significantly lower than tension obtained in the control group [8].

However, few studies have investigated to compare effects of plantar fascia relaxation and self-foam roller according to the posture, on hamstring flexibility. This study was therefore undertaken to investigate the effect of HFRR and sole SMR in sitting and standing positions, on the hamstring flexibility in participants with short hamstrings. We further compared effects of three different release methods (HFRR, sitting SMR and standing SMR) on hamstring flexibility in participants with short hamstrings. We hypothesized that the HFRR, sitting SMR and standing SMR would alter the hamstring flexibility in participants with short hamstrings.

\section{Methods}

\section{Participants}

We applied the G-power software for power analyses (G-power software 3.1.2; Franz Faul, University of Kiel, Kiel, Germany). The necessary sample size of 7 participants was calculated from data obtained from a pilot study of 12 participants, to achieve a power of .80 and an effect size of .55 (Calculated from the partial $\eta 2$ of .23 from the pilot study), with an a level of .05. Thus, we enrolled 20 male participants in this study, with an average age of $23.34 \pm 1.15$ years. The average parameters of all participants were: height $174.95 \pm 5.45 \mathrm{~cm}$, weight 71.58 $\pm 9.37 \mathrm{~kg}$, and body mass index $23.34 \pm 2.52$. The dominant leg was 1 left leg (5\%) and 19 right legs (95\%), and the measured leg was 10 left legs (50\%) and 10 right legs $(50 \%)$. The inclusion criterion for study participation was Active Knee Extension test (AKET) less than $60^{\circ}$. The exclusion criteria included: (1) history of leg pain and lower back pain or injury within six months, (2) symptoms of paralysis or tingling in the lower extremities, and (3) history of surgery on the legs. Prior to the study, all participants provided written informed consent after being explained the entire procedure by the principal investigator. The investigation was approved by the Baekseok University Institutional Review Board (BUIRB: 202003-HR-002).

\section{Measurement and device}

\section{1) Passive knee extension test (PKET) and AKET}

A goniometer was used to measure the angle of PKET and AKET (Goniometer, Fabrication Enterprises Inc, USA). When measuring the PKET, participants were asked to be in supine position on a flat bed, with $90^{\circ}$ flexion 


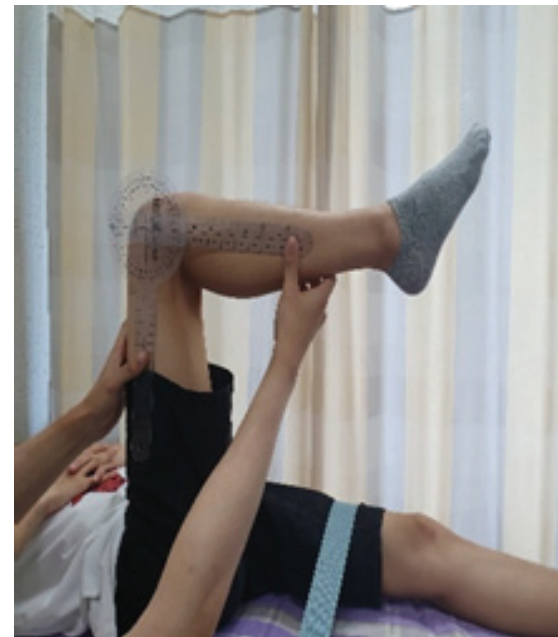

A: start position

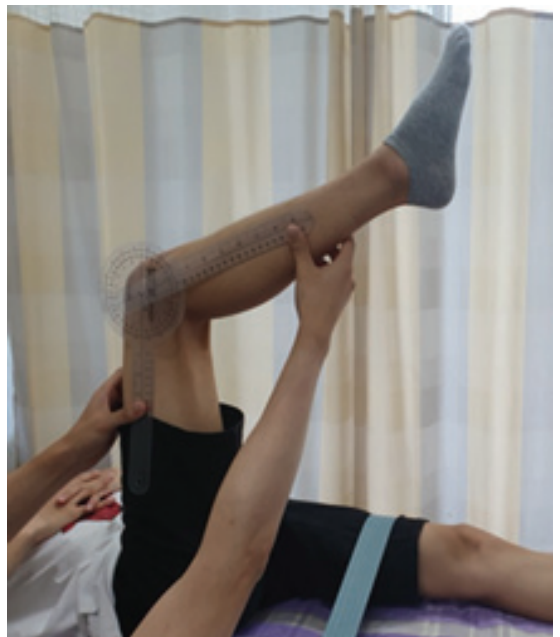

B: end position

Fig. 1. Measurement of active knee extension test.

of hip and knee of the measuring leg. The non-tested leg was kept in an extended position and belted to prevent movement. The axis was aligned in the lateral femoral condyle, the stationary arm in the greater trochanter, and the moving arm in the lateral malleolus direction. In this position, the examiner passively extended the knee until the subject experienced strong resistance. The angle of the final position was subsequently measured $(\mathrm{r}=.96)$ [9]. For measuring the AKET, the same position was maintained as the PKET. However, participants were measured by keeping their knee extension actively for 2-3 seconds, until they felt strong resistance. The intra-class correlation coefficient (ICC) of the AKET measurements obtained were $.87-.94$ (Fig. 1) [10].

\section{2) Finger To Floor distance Test (FTFT)}

The FTFT was applied to measure the mobility of both the whole spine and the pelvis, in an overall motion of bending forward [11]. The participants removed their shoes and stood erect on a $20-\mathrm{cm}$ high wooden box, with feet together and big toe at the end of the box. The second toe and heel were then placed in a straight line. Participants were asked to bend forward as far as possible, while maintaining the knees, arms, and fingers fully extended. The vertical distance between the tip of the middle finger and the top of the box was measured (this refers to the side where the sole of the foot touches) and is expressed in centimeters. The vertical distance between the box and tip of the middle finger was considered positive when the subject did not reach the floor, and negative when he could go further. Both investigators were experienced with measurement of the finger-to-floor distance. Inter-measurement reliability was reported as very high, with an ICC $=.99$ [11].

\section{3) Measurement of Pelvic Tilting Angle (PTAT)}

The Image $\mathrm{J}$ software (Image $\mathrm{J}$ program, National Institutes of Health, USA) was used to measure PTAT. First, a $2 \mathrm{~cm}$ diameter sticker was attached to the ASIS and PSIS on the tested leg. Next, height of the camera lens was adjusted to match the height of the participant's ASIS and was leveled with the floor. Subsequently, imaging was done $1 \mathrm{~m}$ away from sagittal plane of the participant. During imaging, the participants were relaxed, and both feet were placed parallel to the shoulder width, and the gaze was directed to the front. This photograph was used to create virtual lines in ASIS and PSIS using the Image 


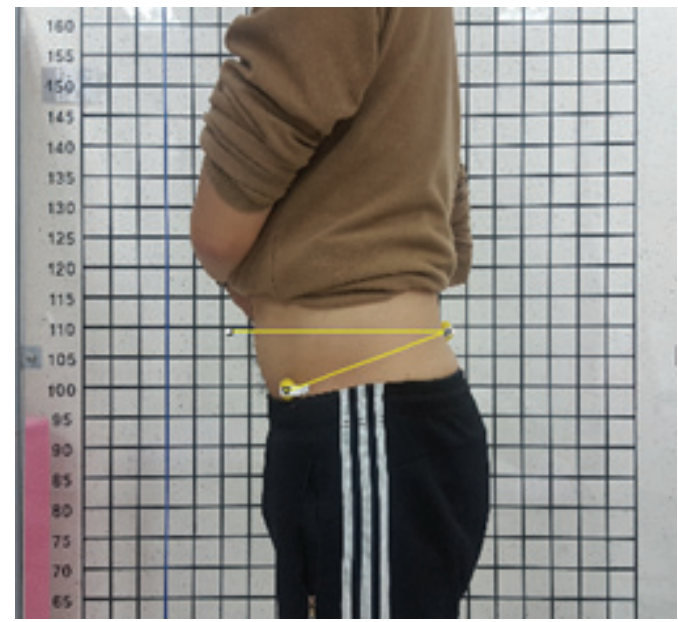

Fig. 2. Measurement of pelvic tilting angle.

$\mathrm{J}$ program. By making and connecting a parallel line with the floor, the angle between the two lines was then measured. Intra-rater reliability obtained was $r=.86[12]$ (Fig. 2).

\section{Intervention}

\section{1) Hamstrings Foam Roller Release (HFRR)}

A standardized foam rolling procedure was followed by the principal investigator. Participants were first asked to lie in a long sitting position, after which they were asked to roll a foam roller (Redbalance, Korea) across the entire hamstring muscle, from the ischial tuberosity to the back of the knee, on the side being examined. The hands supported the floor and did not move during the foam roller exercise. Participants were asked to maintain the maximum weight on the tested leg. The HFRR was performed for 4 sets: exercise 30 seconds + resting 30 seconds [13] (Fig. 3).

\section{2) Sitting Self Myofascial Release (Sitting SMR)}

The participants were initially asked to assume a sitting position. They were then instructed to roll the massage ball (Ausports, South Korea) on the sole of each tested foot, from behind the metatarsal heads to the heel

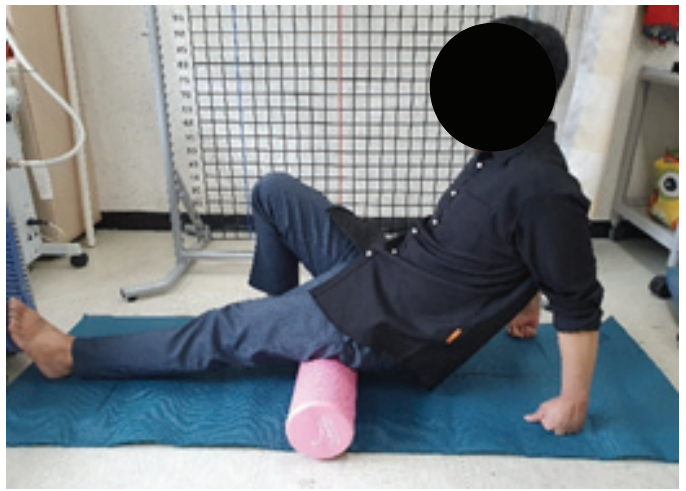

Fig. 3. Hamstring foam roller.

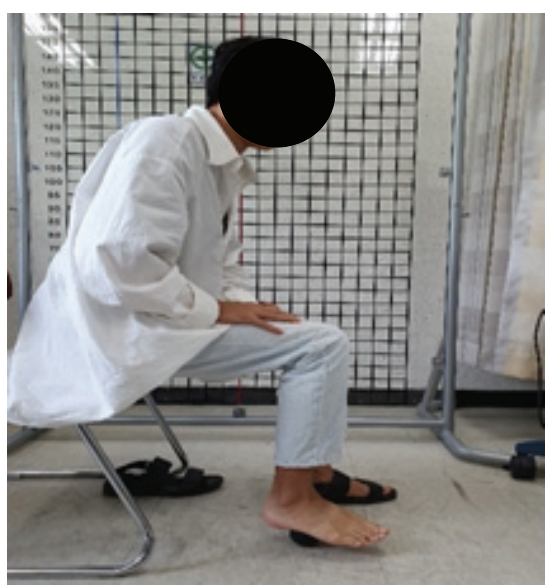

Fig. 4. Sitting self myofascial releases.

concentrating on the medial arch. Participants were instructed to feel discomfort but no pain, by applying as much pressure as possible to the soles, as greater pressure has been shown to increase flexibility [10]. The sitting SMR was performed for 4 sets: exercise 30 seconds + resting 30 seconds (Fig. 4).

\section{3) Standing Self Myofascial Release (Standing SMR)}

Participants were asked to stand upright on a flat floor and use the massage ball (Ausports, South Korea) on the arch of the tested side. Participants were instructed to feel discomfort but no pain, by applying as much pressure as possible to the soles, as greater pressure is shown to increase 
flexibility [14]. The standing SMR was performed for 4 sets: exercise 30 seconds + resting 30 seconds.

\section{Experimental procedure}

Before subjecting to interventions, the AKET was performed to screen participants with short hamstrings. Subjects with short hamstrings were selected if the knee extension angle was less than $60^{\circ}$ in AKET [15]. If both hamstrings were short, the experiment was performed by selecting the leg with the smaller AKET angle. The results were not recorded by any investigator, but values of the results were read by a student so that the investigator remained blinded. Before intervention, measurements were taken in the order PKET, AKET, FTFT, and PTAT, with a one minute rest taken between each measurement; the mean value was then used for data analysis. Participants performed the three interventions in randomized order; subjects drew lots to avoid learning effects or fatigue. The resting time between interventions was 48 hours to 120 hours, to avoid the effects of previous interventions [13]. Participants were familiarized with the exercises prior to testing. The familiarization period ended when the participant was able to maintain the exercise position for 5 seconds. All participants were comfortable after the familiarization period, and none reported fatigue. A 5 minutes rest period was allowed after the familiarization period and before data collection began. The PKET, AKET, FTFT, and PTAT were measured immediately after each intervention. If the participant felt severe pain during the procedure, the experiment was stopped and excluded from the analysis.

\section{Statistical analysis}

The collected data were analyzed using the IBM SPSS Statistics ver. 20.0 (IBM Co., New York, USA) statistical program for Windows. General characteristics of the participant were used in the descriptive statistics. Normality test was performed using the Kolmogorov-Smirnov test.
The data were normally distributed, and the parameter test was performed. Paired t-tests were used to determine the differences before and after interventions, and one-way ANOVA was used to compare post values among the three interventions. The significance level for testing statistical significance was set to $a=05$.

\section{Results}

\section{Comparison of pre and post interventions}

Compared to pre-intervention, the PKET, AKET, and FTFT values significantly increased after interventions ( $p$ $<.05)$. However, PTAT values showed no significant difference between pre and post interventions for all three procedures $(p>.05)$ (Table 1).

\section{Comparisons among the interventions}

The PKET, AKET, FTFT, and PTAT values did not differ significantly among the three interventions $(p>.05)$ (Table 2).

\section{Discussion}

This study was undertaken to investigate the effect of HFRR and sole SMR based on anatomy trains, on hamstring flexibility. Our results indicate that PKA, AKET and FTFT values increase significantly in three interventions, with no significant difference in the comparison between the interventions.

PKET, AKET and FTFT were significantly increased after HFRR $(20.3 \%, 23.21 \%$, and $52.31 \%$, respectively). According to a previous study, the HFRR in healthy adults significantly increased the PKET compared to before intervention [16]. The mechanism of foam rollers to increase the ROM is due to direct pressure on the soft tissue during rolling, which directly stretches the muscles and myofascia [17]. In addition, the friction created during the rolling exercise raises the temperature of the myofascia, 
Table 1. Comparisons of PKET, AKET, FTFT, and PTAT before and after the Three Interventions $(n=20)$

\begin{tabular}{ccccccc}
\hline Variables (Unit) & Exercises & Pre Test & Post Test & Differences & $\mathrm{t}$ & $\mathrm{P}$ \\
\hline \multirow{2}{*}{ PKET $\left(^{\circ}\right)$} & HFRR & & $52.75 \pm 10.44$ & $8.75 \pm 6.17$ & -6.340 & $.000^{*}$ \\
& Sitting SMR & $44.00 \pm 11.02^{\mathrm{a}}$ & $57.35 \pm 10.64$ & $13.35 \pm 8.21$ & -7.272 & $.000^{*}$ \\
& Standing SMR & & $54.00 \pm 14.29$ & $10.00 \pm 8.93$ & -5.007 & $.000^{*}$ \\
\hline & HFRR & & $48.25 \pm 10.74$ & $8.25 \pm 6.41$ & -5.752 & $.000^{*}$ \\
AKET $\left(^{\circ}\right)$ & Sitting SMR & $40.00 \pm 10.21$ & $50.95 \pm 9.74$ & $10.95 \pm 9.72$ & -5.038 & $.000^{*}$ \\
& Standing SMR & & $51.00 \pm 10.28$ & $11.00 \pm 7.03$ & -6.994 & $.000^{*}$ \\
\hline & HFRR & & $-3.60 \pm 10.69$ & $4.15 \pm 2.77$ & -6.683 & $.000^{*}$ \\
FTFT $(\mathrm{cm})$ & Sitting SMR & $-7.75 \pm 11.23$ & $-3.90 \pm 11.33$ & $3.85 \pm 4.24$ & -4.055 & $.001^{*}$ \\
& Standing SMR & & $-3.10 \pm 11.46$ & $4.65 \pm 4.49$ & -4.622 & $.000^{*}$ \\
\hline & HFRR & & $11.15 \pm 3.66$ & $.30 \pm 3.09$ & -.433 & .670 \\
PTAT $\left({ }^{\circ}\right)$ & Sitting SMR & $10.85 \pm 3.76$ & $9.85 \pm 4.22$ & $1.00 \pm 2.57$ & 1.737 & .099 \\
& Standing SMR & & $11.15 \pm 3.54$ & $.30 \pm 2.95$ & -.454 & .655 \\
\hline
\end{tabular}

${ }^{a}$ Mean \pm standard deviation, ${ }^{*} p<.05$

PKET: passive knee extension test; AKET: active knee extension test; FTFT: finger to floor distance test; PTAT: pelvic tilting angle test; HFRR: hamstring form roller release; sitting SMR: sitting self myofascial releases; standing SMR: standing self myofascial releases.

Table 2. Comparisons of Post Value of the Three Interventions PKET, AKET, FTFT, PTAT $(n=20)$

\begin{tabular}{cccccc}
\hline & HFRR & Sitting SMR & Standing SMR & F & P \\
\hline PKET $\left(^{\circ}\right)$ & $52.75 \pm 10.44^{\mathrm{a}}$ & $57.35 \pm 10.64$ & $54.00 \pm 14.29$ & 2.881 & .082 \\
AKET $\left({ }^{\circ}\right)$ & $48.25 \pm 10.74$ & $50.95 \pm 9.74$ & $51.00 \pm 10.28$ & 1.671 & .216 \\
FTFT $(\mathrm{cm})$ & $-3.60 \pm 10.69$ & $-3.90 \pm 11.33$ & $-3.10 \pm 11.46$ & .455 & .642 \\
PTAT $\left({ }^{\circ}\right)$ & $11.15 \pm 3.66$ & $9.85 \pm 4.22$ & $11.15 \pm 3.54$ & 1.602 & .229 \\
\hline
\end{tabular}

${ }^{\mathrm{a}}$ Mean \pm standard deviation

PKET: passive knee extension test; AKET: active knee extension test; FTFT: finger to floor distance test; PTAT: pelvic tilting angle test; HFRR: hamstring form roller release; sitting SMR: sitting self myofascial releases; standing SMR: standing self myofascial releases.

which breaks the fibrous adhesions between different layers of the fascia and changes the flexible gel form, thereby relaxing the soft tissue [18]. In the current study, the same principle applies when performing HFRR, resulting in increased PKET, AKET, and FTFT, by relaxation of the hamstring muscles and myofascia. Therefore, as per results achieved in this study, the HFRR would be a useful method to improve flexibility of the hamstrings in subjects with short hamstring.

The PKET, AKET, and FTFT were significantly increased after sitting SMR and standing SMR (Sitting SMR: $20.3 \%, 27.42 \%$, and 48.08\%, respectively; standing SMR: $23.04 \%, 27.55 \%$, and $60.36 \%$, respectively). According to a previous study, sitting on a healthy adult and performing sole SMR significantly increased flexibility of the hamstrings through sit-and-reach, as compared to the control group [19]. Another study reported that the tension of erector spinae decreased significantly when the myofascia was relaxed by sole stretching [8]. Recent studies have shown that the sole ball massage by the therapist 
in patients with short hamstrings significantly increased the flexibility (PKET and Sit-and-Reach Test) of the hamstrings, compared to before intervention [20]. These results can be explained on the basis of the schematic map (Anatomic map) and tensegrity principle. The tensegrity principle emphasizes the presence of continuity and connectivity between anatomically distant fasciae or muscles [21,22], and a schematic map underlines that tension in certain areas has a detrimental effect on the overall flexibility [6]. Therefore, in this study, we postulated that flexibility of the hamstrings would be improved by applying sole SMR in subjects with short hamstring.

The PTAT was not significantly different between the three interventions, and between pre and post intervention. This study hypothesized that myofascial relaxation would change the pelvic tilt angle, based on the theory of "superficial back line". The "superficial back line" theory consists of the plantar fascia, short toe flexor (Lumbricals, flexor accessorius and flexor digitorum brevis), Achilles tendon, calf muscles (Gastrocnemius and soleus), hamstrings (Semimembranosus, semitendinosus and biceps femoris), sacrotuberous ligament, fascia of the sacrolumbar area, erector spinae, and finally the epicranial fascia [6]. However, according to other previous research, Fasuyi FO et al. [23] reported that subjects without lower back pain had significantly longer hamstring length compared to participants with lower back pain, and the pelvic tilt angle was not significantly different. However, Król A et al. [24] found that the relationship between hamstring length and lower back pain was not significant, and that the length did not significantly affect the pelvic tilt. Therefore, results of the hamstrings length and pelvic tilting angle are controversial, and further studies are required in this area.

The treatment pressure depends on the force and area of contact applied by the foam roller or massage ball. This study was conducted on the hypothesis that there would be significant differences between the three interventions.
However, no significant differences were obtained in the PKET, AKET, FTFT, and PTAT values, when compared among the interventions. It is therefore suggested that HFRR, sitting SMR and standing SMR can be helpful to increase the hamstring flexibility in subjects with short hamstring.

This study has several limitations. First, its generalizability is limited since young male participants with no symptoms of lower back pain were the participant subjects. The results may have been different with female participants or participants with such symptoms were involved. Second, this study was to investigate the immediate effect. Therefore, long-term effects of the three release methods cannot be determined. Further studies should compare the long-term effects of these different methods in participants with short hamstrings. Third, although the three interventions were performed in a random order, it seems that the validity of the study results is probably low, because four tests were sequentially performed on the same subject without a control group. Therefore, it is necessary to randomly proceed with four tests in future studies.

\section{Conclusion}

This study investigated immediate changes in the flexibility of hamstrings, by applying HFRR and massage ball on the sole. All three interventions showed significant changes before and after intervention in PKET, AKET and FTFT, but no significant change in PTAT. No significant differences were obtained among the three methods. Therefore, we propose that the HFRR, sitting SMR and standing SMR are immediately effective in improving hamstring flexibility in participants with short hamstrings.

\section{References}

[1] Kim GC, Lee JH, Kwon SM. Effects of hamstring flexibility and dynamic stability of lower lumbar according 
to stretching and massage techniques. Journal of Korean Society of Physical Medicine. 2013;8(4):609-17.

[2] Neumann DA. Kinesiology of the musculoskeletal system-e-book: foundations for rehabilitation. Elsevier Health Sciences. 2013.

[3] Kim A, Kwon J, Lee H. The Effect of Static Stretching Loading on Hamstring Flexibility in Healthy individuals. The Korean Society of Sports Science. 2015;24:1341-8.

[4] Mohr AR, Long BC, Goad CL. Effect of foam rolling and static stretching on passive hip-flexion range of motion. Journal of sport rehabilitation. 2014;23(4):296-9.

[5] Schleip R, Jäger H, Klingler W. What is 'fascia'? A review of different nomenclatures. Journal of bodywork and movement therapies. 2012;16(4):496-502.

[6] Myers TW. Anatomy trains e-book: myofascial meridians for manual and movement therapists. Elsevier Health Sciences. 2013.

[7] Jung J. Analysis for Myofascia meridian effects on Sports Biomechanics characteristics at Taekwondo side kick in elete players. Konkuk University. 2017;Master's Degree.

[8] Shin D, Shin H, Chung S. The Effect of Plantar Fascia Stretching on the Tension of Erector Spinae Muscle and Hamstring Muscle. J Oriental Rehab Med. 2005;15(4): 29-38.

[9] Nelson RT, Bandy WD. Eccentric training and static stretching improve hamstring flexibility of high school males. Journal of athletic training. 2004;39(3):254.

[10] Neto T, Jacobsohn L, Carita AI, et al. Reliability of the active-knee-extension and straight-leg-raise tests in subjects with flexibility deficits. Journal of sport rehabilitation. 2015;24(4).

[11] Perret C, Poiraudeau S, Fermanian J, et al. Validity, reliability, and responsiveness of the fingertip-to-floor test. Archives of physical medicine and rehabilitation. 2001;82(11):1566-70.

[12] Kim D, Shim J, Choung S. The Effect of Deep Friction Massage, Modified Thomas's Stretching and Muscle Energy Technique on Thickness of Psoas Major and Pelvic
Angle. Archives of Orthopedic and Sports Physical Therapy. 2016;12(2):1-7.

[13] Couture G, Karlik D, Glass SC, et al. The effect of foam rolling duration on hamstring range of motion. The open orthopaedics journal. 2015;9:450.

[14] Williams W, Selkow NM. Self-myofascial release of the superficial back line improves sit-and-reach distance. Journal of sport rehabilitation. 2019;29(4):400-4.

[15] Bandy WD, Irion JM, Briggler M. The effect of time and frequency of static stretching on flexibility of the hamstring muscles. Physical therapy. 1997;77(10): 1090-6.

[16] Lee M. Comparison of the Self-Myofascial Release Technique and the Graston Technique in an Adult Male's flexibility. Korea national sport University. 2017;Master's Degree.

[17] MacDonald GZ, Penney MD, Mullaley ME, et al. An acute bout of self-myofascial release increases range of motion without a subsequent decrease in muscle activation or force. The Journal of Strength \& Conditioning Research. 2013;27(3):812-21.

[18] Curran PF, Fiore RD, Crisco JJ. A comparison of the pressure exerted on soft tissue by 2 myofascial rollers. Journal of sport rehabilitation. 2008;17(4):432-42.

[19] Grieve R, Goodwin F, Alfaki M, et al. The immediate effect of bilateral self myofascial release on the plantar surface of the feet on hamstring and lumbar spine flexibility: a pilot randomised controlled trial. Journal of bodywork and movement therapies. 2015;19(3):544-52.

[20] Joshi DG, Balthillaya G, Prabhu A. Effect of remote myofascial release on hamstring flexibility in asymptomatic individuals-A randomized clinical trial. Journal of bodywork and movement therapies. 2018;22(3):832-7.

[21] Langevin HM. Connective tissue: a body-wide signaling network? Medical hypotheses. 2006;66(6):1074-7.

[22] Kassolik K, Jaskólska A, Kisiel-Sajewicz K, et al. Tensegrity principle in massage demonstrated by electro-and mechanomyography. Journal of Bodywork and Movement 
Therapies. 2009;13(2):164-70.

[23] Fasuyi FO, Fabunmi AA, Adegoke BO. Hamstring muscle length and pelvic tilt range among individuals with and without low back pain. Journal of Bodywork and Movement Therapies. 2017;21(2):246-50.
[24] Król A, Polak M, Szczygieł E, et al. Relationship between mechanical factors and pelvic tilt in adults with and without low back pain. Journal of back and musculoskeletal rehabilitation. 2017;30(4):699-705. 
